\title{
High Density Perforation of Thin Al-Foils with Ultra Short Pulse Lasers
}

\author{
Claudia HARTMANN ${ }^{* 1}$, Nelli HAMBACH ${ }^{* 1}$, Michael JÜNGST ${ }^{* 1}$, Stephan KELLER ${ }^{* 1}$, Jens HOLTKAMP ${ }^{* 1}$ \\ and Arnold GILLNER ${ }^{*}$ \\ ${ }^{*}$ Fraunhofer Institute for Laser Technology ILT, Steinbachstr. 15, 52074 Aachen, Germany \\ E-mail: Claudia.Hartmann@ilt.fraunhofer.de
}

\begin{abstract}
The need for micro scaled features in medical devices and engineering products, like holes for filtration, separation and ventilation or small structures e.g. for hydrophobic effects, is constantly increasing. The size of these structures is often in the region of $20 \mu \mathrm{m}$ and smaller. One important aspect of the drilling or structuring process is the process stability and its influencing factors. For the laser perforation of a $15 \mu \mathrm{m}$ thick Aluminum foil with UV ps-pulses the process limits as well as the influencing factors are investigated. The pitch of the holes within the pattern as well as the number of pulses used for the percussion drilling of each of the holes are varied. The drilled holes are evaluated by means of hole diameter and precision of the hole shape in means of circularity and ellipticity. Holes with a diameter of $<6 \mu \mathrm{m}$ are drilled at a minimal pitch of $12 \mu \mathrm{m}$. The ellipticity is mainly dependent on the heat distribution, the circularity on polarization effects. In contrast to the perforation process with ns-pulses polarization effects play a major role for the perforation process with ps-pulses.
\end{abstract}

DOI: $10.2961 / \mathrm{jlmn} .2013 .03 .0013$

Keywords: laser drilling, perforation, ps-laser, thin foil drilling, stability limits

\section{Introduction}

Laser micro drilling and micro structuring is used for many applications in areas such as filtration, electronics, sensor systems, medicine but also in the tooling industry [1]. For the establishment of the laser ablation technique in these industries a fast, precise and reproducible process is needed [2].

During the last years ps-lasers have been established more and more for industry applications [3, 4, 5]. Due to the short pulse duration the heat affected zone is reduced. But still thermal effects can occur depending on the machining parameters $[6,7]$.

These thermal effects as well as other machine and process influences limit the stability of the laser processes. As many parts such as filters or injection molds can have very large areas which have to be drilled or structured, the laser process needs to be constant for hours or even days [2].

For the drilling of $50 \mu \mathrm{m}$ Aluminum foils with UV nspulses the main influencing factors with regards to the stability of the perforation process have been identified. In this study holes with a diameter of $20 \mu \mathrm{m}$ or smaller have been investigated. One limiting factor for the perforation process is the ellipticity of the holes. The main influencing effects have been identified to be thermal effects in combination with the drilling strategy as well as the stability of the laser source itself [8].

Within the here presented work the influencing effects on the process stability of micro machining with ps-pulses is investigated based on the perforation process of Aluminum foil. A $15 \mu \mathrm{m}$ thick foil is perforated with UV ps-pulses. The perforated area is drilled in jump and shoot mode, each hole is produced by percussion drilling. The aim is to achieve round and uniform-sized holes on large areas.

\section{Experimental Setup}

The experiments are carried out with a diode pumped $\mathrm{Nd}: \mathrm{YVO}_{4}$ MOPA laser (Master Oscillator Power Amplifier; Hyper Rapid, LumeraLaser) with three amplification stages at a wavelength of $\lambda=355 \mathrm{~nm}$. The laser operates at a maximum average power of $P=20 \mathrm{~W}$ at a repetition rate up to $v=1 \mathrm{MHz}$ and a pulse duration of $\tau<15 \mathrm{ps}$. The average power can be attenuated internally. For the experiments the frequency is set to $v=400 \mathrm{kHz}$, the average power is adjusted between $P=0,3$ and $0,7 \mathrm{~W}$.

The laser beam is moved by a galvanometric scanner (intelliSCANde ${ }^{\circledR} 14$, SCANLAB) and focused on the foil surface by a telecentric f- $\Theta$-lens with focal length $f=56 \mathrm{~mm}$. The focus diameter is measured to $d=10 \mu \mathrm{m}$ at a Rayleigh length of $z_{\mathrm{R}}=230 \mu \mathrm{m}$. The sample material is $15 \mu \mathrm{m}$ thick Al foil which is uniformly stretched by a clamping device and positioned with linear axes.

The drilled holes are conical with bigger entrance diameter than exit diameter. For most applications the maximal transmission, meaning the maximal removed area, of the foil is of interest. Therefore the holes are measured at the smallest diameters. For measurement of the diameter SEM pictures from top side are made.

Within the experiments triangular hole patterns are drilled. The hole patterns consists of $>3000$ holes. Each hole is drilled by percussion drilling with $n=100$ to 250 pulses per hole. The distance between the center of two adjacent holes is called pitch $a$, see Figure 1. During the experiments the pitch has been varied between $a=12$ and $50 \mu \mathrm{m}$. 


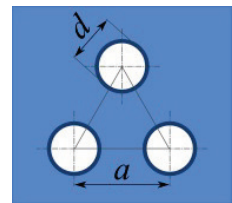

Figure 1: Triangular hole pattern. $a$ : pitch and $d$ : diameter of the holes.

The position and drilling order of all holes in the pattern is defined in a coordinate list which is transferred to the scanner. Two drilling orders are investigated: "in-line" and "random", see Figure 2. For the "in-line" drilling strategy both the $\mathrm{x}$ - and the $\mathrm{y}$-coordinates are sorted in ascending order (Figure 2 a). For the "random" drilling strategy the coordinates in the list are mixed randomly (Figure $2 \mathrm{~b}$ ). For both drilling strategies the coordinate lists are generated only once and afterwards the same lists resp. the order of the holes within the list is used for all tests. Therefore for all tests the order of the holes drilled with the same strategy remains constant.
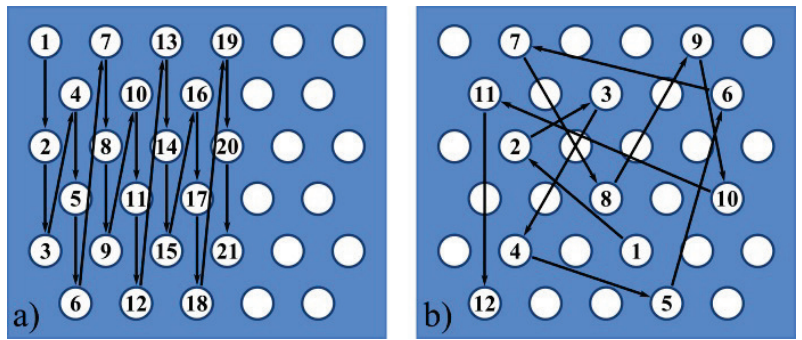

Figure 2: Investigated drilling strategies. a) "in-line" and b) "random".

In the here presented work two measurement series are presented. The parameters varied in these experiments are listed in Table 1.

Table 1: parameters varied in the experiments presented in this paper.

\begin{tabular}{lcc}
\hline Parameter & variation pitch & $\begin{array}{c}\text { variation number } \\
\text { of pulses }\end{array}$ \\
\hline Strategy & random / in-line & random \\
Number of pulses & 160 & $75-250$ \\
Pitch & $10-25 \mu \mathrm{m}$ & $50 \mu \mathrm{m}$ \\
Pulse energy & $1.8 \mu \mathrm{J}$ & $0,75 \mu \mathrm{J}$ \\
Repetition rate & $400 \mathrm{kHz}$ & $400 \mathrm{kHz}$ \\
\hline
\end{tabular}

For evaluation of the drilling result the shape of the holes is evaluated. Therefore the diameter of the holes in maximal and minimal dimension is measured, see Figure 3.

Both dimensions are of interest as they define different aspects. For example filtration processes need a defined filtration rate at a maximal perforation degree. The filtration rate is defined by the minimal diameter of the structures as this diameter defines the larges particle size that is hold back. At the same time enlarges a bigger maximal diameter the perforation degree as more material is removed.

For evaluation of the shape of the holes two different parameters are defined: the circularity $\gamma=\frac{4 \pi A}{U^{2}}$ and the ellipticity $\varepsilon=\frac{d_{\min }}{d_{\max }} . A$ is the measured area of the hole and $U$ the measured circumference, see Figure 3.

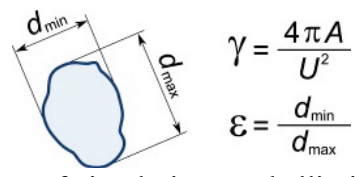

Figure 3: Definition of circularity $\gamma$ and ellipticity $\varepsilon$ of the holes $d_{\text {min }}$ : minimal diameter, $d_{\max }$ : maximal diameter, $A$ : area of the hole and $U$ : circumference of the hole

By defining these two characteristics of the shape of the hole two different deviations from a round shape of the holes can be seen. The circularity $\gamma$ changes mainly when the circumference of the hole is not smooth but does not take account for the overall geometry of the hole. The ellipticity $\varepsilon$ only takes account for the overall geometry and not for the circumference.

The perforation degree $\xi$ is defined as the ratio of the drilled area to the sample area. For a triangular hole pattern the perforation degree is given by:

$$
\xi=\frac{A}{2 \sqrt{3} a^{2}}
$$

$A$ is the measured hole area and $a$ the pitch, see Figure 1. The maximal perforation degree $\xi_{\max }$ (given by the minimal pitch) defines the maximal area which can be drilled without destroying the sample.

\section{Results and Discussion}

Perforated areas are produced with varied pitch for both drilling strategies. The number of pulses per holes is set to $\mathrm{n}=160$, the pulse energy to $E=1.8 \mu \mathrm{J}$.

Minimal and maximal diameter of the holes as a function of the pitch as well as circularity and ellipticity are investigated. For in-line strategy perforated areas with a minimal pitch $a=12 \mu \mathrm{m}$ can be produced before the foil tears, see Figure 4. For random strategy the minimal pitch is $a=13 \mu \mathrm{m}$, see Figure 5 .

For both drilling strategies the maximal diameter of the holes is bigger for pitches $a<14 \mu \mathrm{m}$ than for larger pitches $a>15 \mu \mathrm{m}$. The minimal diameter is nearly constant for all pitches.

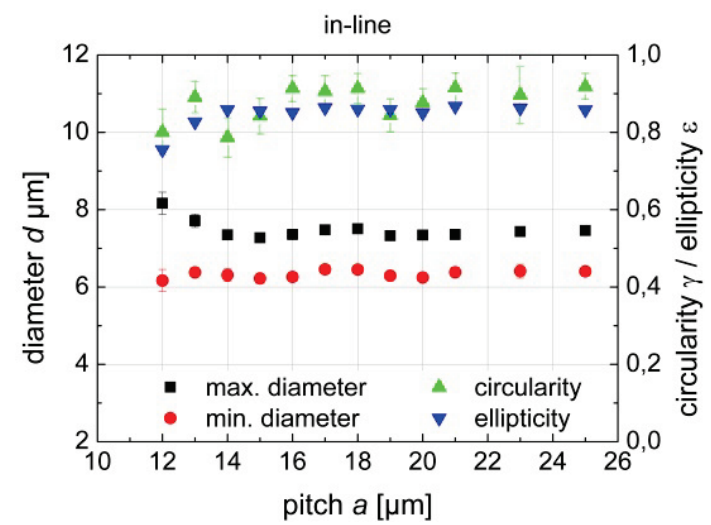

Figure 4: Minimal and maximal diameter of the drilled holes as well as circularity and ellipticity for the variation of the pitch. (n $=160, E=1.8 \mu \mathrm{J}, v=400 \mathrm{kHz}$, drilling strategy: in-line) 


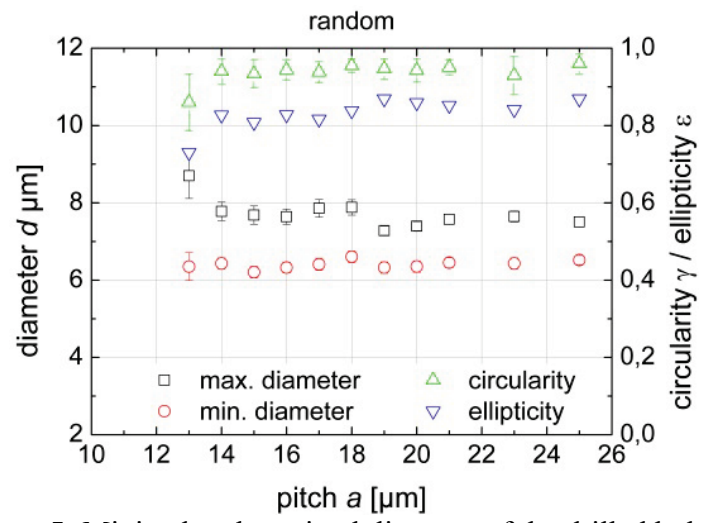

Figure 5: Minimal and maximal diameter of the drilled holes as well as circularity and ellipticity for the variation of the pitch. (n $=160, E=1.8 \mu \mathrm{J}, v=400 \mathrm{kHz}$, drilling strategy: random)

This leads to a smaller ellipticity for $a<14 \mu \mathrm{m}$. Here the holes influence one another that the shape changes to be more elliptic, see Figure 4 and Figure 5. The behavior of the ellipticity $\varepsilon$ as a function of the pitch is different for inline and random strategy. For in-line strategy the ellipticity for $a>15 \mu \mathrm{m}$ is very constant (Figure 5), for random strategy the ellipticity varies by ca. $10 \%$ for pitches $a<$ $19 \mu \mathrm{m}$ and is constant for bigger pitches $a \geq 19 \mu \mathrm{m}$ (Figure 4).

For in-line strategy the circularity of the holes varies more than $10 \%$ with changing pitch. Tendentially the circularity gets better (larger values) with increasing pitch, see Figure 4. For random strategy the circularity for $a>$ $14 \mu \mathrm{m}$ is nearly constant, see Figure 5 .

The area of the holes for in-line strategy is $A=38 \mu \mathrm{m}^{2}$ for a pitch of $a=12 \mu \mathrm{m}$ and $A=34,2 \mu \mathrm{m}$ for $a=14 \mu \mathrm{m}$. For random drilling strategy the area of the holes is $A=$ $42,2 \mu \mathrm{m}^{2}$ for a pitch of $a=13 \mu \mathrm{m}$ and $A=39 \mu \mathrm{m}$ for $a=$ $14 \mu \mathrm{m}$. This leads to a maximal perforation degree of $\xi=$ $7,6 \%$ for in-line strategy and 7,2\% for random strategy. At $a=14 \mu \mathrm{m}$ the perforation degree is $\xi=5 \%$ for in-line strategy and 5,7\% for random strategy.

These results lead to several conclusions:

- Process for in-line strategy is more stable than for random strategy as the pitch can be chosen smaller before the foil tears.

- The thermal influence on the drilling process is especially evident for pitches $a<14 \mu \mathrm{m}$ as here the holes become extremely elliptical.

- The smallest pitch for a stable process is a = $14 \mu \mathrm{m}$, at lower pitches the holes get too elliptical. At this pitch a perforation degree of $\xi=5,7 \%$ for random strategy and $\xi=5 \%$ for in-line strategy is achieved.

- The circularity $\gamma$ and the ellipticity $\varepsilon$ have different sources as they behave differently for the two drilling strategies (in-line: ellipticity is constant, random: circularity is constant)

There are two main differences in the two drilling strategies for the drilling process: the direction of heat propagation for each drilled hole and the process time. The process time for in-line strategy is shorter than for random strategy as the jump distance between two drilled holes is much smaller. Therefore the foil is not only drilled directly next to the just heated material but also has less time to cool down between drilling of the holes.

In the SEM pictures of the drilled patterns for in-line and random strategy these additional effects shall be defined.

For pitches $a \geq 19 \mu \mathrm{m}$ there is no interference of the melt with already drilled holes in the pattern, see Figure 6.
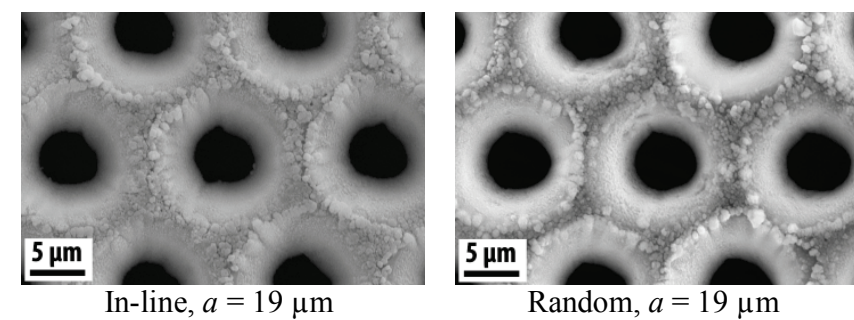

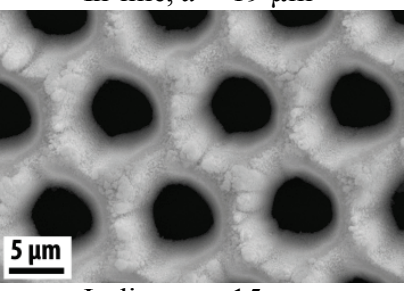

In-line, $a=15 \mu \mathrm{m}$

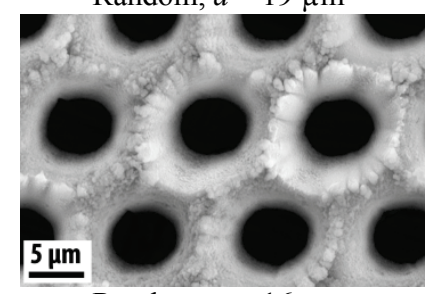

Random, $a=16 \mu \mathrm{m}$
Figure 6: Variation of the pitch for drilling in random and in-line drilling strategy. $(\mathrm{n}=160, E=1.8 \mu \mathrm{J}, v=400 \mathrm{kHz})$

For small pitches $a<19 \mu \mathrm{m}$ the newly drilled holes interfere with the already drilled ones in the pattern. The interference is different for the two drilling strategies.

For in-line strategy the left over material between two adjacent holes which has not cooled down completely is deformed asymmetrically. The material is pushed into the already drilled holes to deform those holes, see Figure 6 for in-line strategy at a pitch of $a=15 \mu \mathrm{m}$. Due to this deformation the taper shape of the drilled holes gets very asymmetrically. Additionally an elliptical deformation of the hole diameter is caused which is very uniform. But the smoothness of the hole shape is not uniform due to deformation which leads to a variation of the circularity $\gamma$ especially for smaller pitches $a<20 \mu \mathrm{m}$, see Figure 4 .

For the random drilling strategy for pitches smaller than $a<20 \mu \mathrm{m}$ the melt of the subsequently drilled holes is pushed into the former drilled holes. This is shown for $a=$ $16 \mu \mathrm{m}$ in Figure 6. Due to random drilling strategy the holes drilled consecutively are only rarely positioned next to each other. Therefore the foil around a drilled hole has cooled down before the next hole is drilled and is not deformed during drilling of a following hole. As the material around he holes is not deformed afterwards, the smoothness of the hole shape is also not changed afterwards and the circularity is homogeneous for all pitches despite the smallest, see Figure 5.

The variation of the ellipticity $\varepsilon$ is to account to changes in the heat distribution within the foil for all drilled holes. As for each hole the already drilled pattern changes the heat distribution in which direction the heat can dissipate, the ellipticity varies more than for in-line process.

Next the number of pulses for drilling holes is changed for a constant, wide pitch in random drilling strategy. The pitch is $a=50 \mu \mathrm{m}$, big enough that the holes do not 
interfere with each other. The number of pulses is changed between $\mathrm{n}=100$ and 250 .

For $\mathrm{n} \leq 150$ pulses the minimal and maximal hole diameter increase with increasing number of pulses. For $n$ $\geq 150$ pulses both diameters remain nearly constant, see Figure 7.

The ellipticity $\varepsilon$ is nearly constant for all investigated numbers of pulses. The circularity $\gamma$ is constant for $\mathrm{n} \leq 175$ and decreases (gets worse) with further increasing $n>175$, see Figure 7.

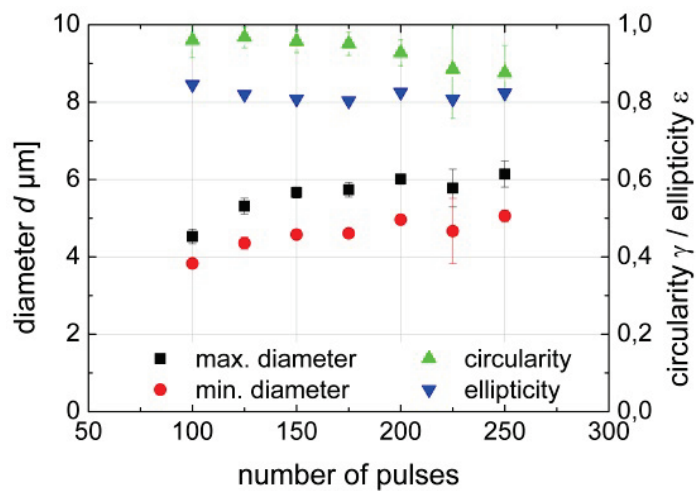

Figure 7: Minimal and maximal diameter of the drilled holes as well as circularity and ellipticity for the variation of the number of pulses for percussion drilling. ( $a=50 \mu \mathrm{m}, E=$ $0.75 \mu \mathrm{J}, v=400 \mathrm{kHz}$, drilling strategy: random)

Summarized this can be interpreted as following, see Figure 7:

- For $\mathrm{n}<150$ pulses the drilling process has not been finished.

- For $\mathrm{n}>175$ pulses the drilling process is completed and parasitic effects start to worsen the drilling result leading to a worse value of the circularity $\gamma$.

- Heat distribution in the foil during drilling of the holes is for all numbers of pulses constant which leads to no change in the ellipticity $\varepsilon$.

- Therefore the process is limited to a small window of roughly $150<\mathrm{n}<175$ pulses per hole to achieve a good and stable drilling result.

Due to the large pitch there is no influence of following drilled holes. The reason for the degration of the circularity SEM pictures of two holes drilled with $\mathrm{n}=150$ pulses and $\mathrm{n}=225$ pulses are shown in Figure 8 .

For larger numbers of pulses per hole a superposed line structure in the taper of the hole can be seen, see Figure 8 right. This effect starts at a number of pulses per hole of $n$ $=175$ pulses.

This additional line structure is aligned perpendicular to the polarization of the used laser beam and increases with increasing number of pulses per hole. In literature $[9,10]$ elliptical bore hole geometries when drilling with linear polarizes lasers has been described for thicker material $(\geq$ $200 \mu \mathrm{m}$ for $[9,10])$. For the drilling of $50 \mu \mathrm{m}$ thick foil no influence of the polarization could be seen [8]. Here is shown the polarization has a major influence on the shape of drilled holes even for such a thin material of $15 \mu \mathrm{m}$ thickness.
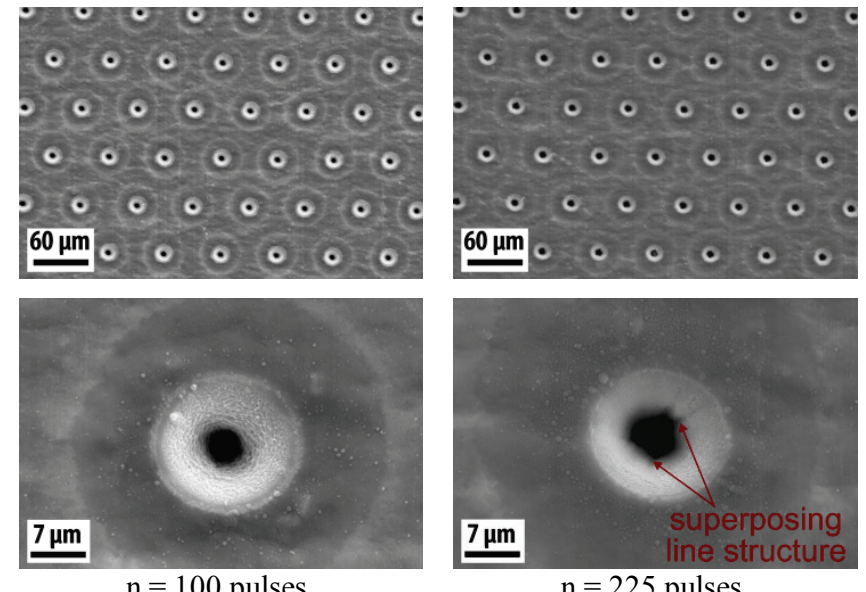

$\mathrm{n}=100$ pulses

$\mathrm{n}=225$ pulses

Figure 8: Variation of number of pulses used for drilling of each hole. $(a=50 \mu \mathrm{m}, E=0.75 \mu \mathrm{J}, v=400 \mathrm{kHz}$, drilling strategy: random)

This shows that already for very thin material, here $15 \mu \mathrm{m}$ thick foils, polarization plays a dominant role for the ablation result. This result is in contrast to the results achieved with ns-pulses where the polarsation has no dominant effect on the shape of the holes [8].

An additional effect can be seen in the SEM picture in Figure 8. Around the taper entrance of the holes a ring structure is formed. This structure has no height which can be measured and is therefore no ablated or deposited material.

When comparing these ring structures to pictures of the foil directly after drilling without any cleaning processes, a similar but blue ring structure around the holes can be seen, see Figure 9.

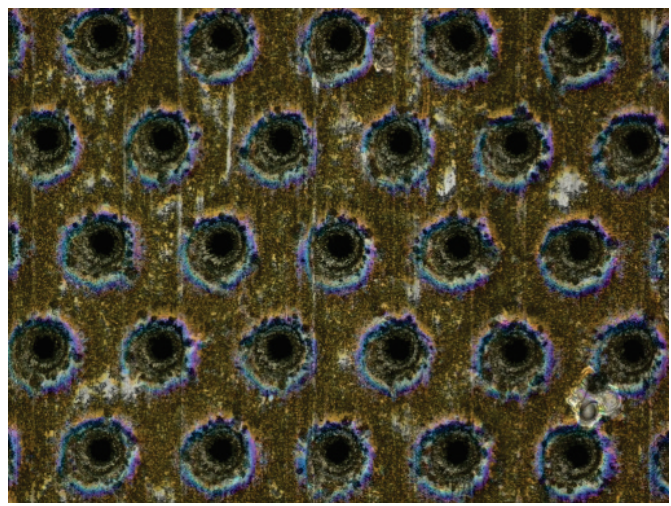

Figure 9: Perforated foil directly after drilling process without cleaning. Around the holes tempering colors can be seen.

After cleaning the ring structure cannot be seen any longer. The source of the rings is assumed to be a thin oxide layer which forms during the perforation process and which is removed during cleaning.

Comparing the here defined limiting factors with the drilling process for ns-lasers [8] different aspects limit the perforation process.

For drilling with ns-lasers the shape of the holes is elliptical, see Figure 10. The ellipticity $\varepsilon$ changes with changing pulse energy and drilling strategy. Polarization as a cause for the ellipticity has been excluded. The main reasons for the ellipticity of the holes are pointing stability and heat propagation in the foil [8]. 


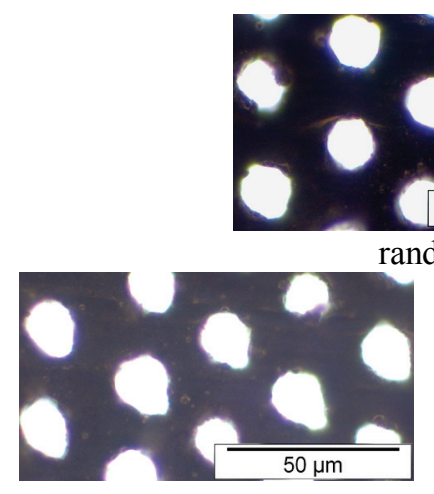

in-line a

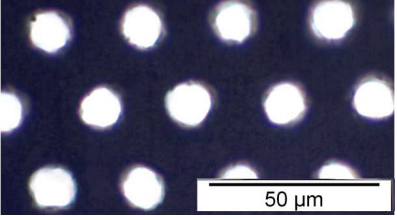

in-line $b$
Figure 10: With ns-pulses perforated Aluminum foil for three different drilling strategies. in-line $a$ and in-line $b$ is the drillin order rotated by $90^{\circ}[8]$

For the drilling with ps-pulses the influence of the drilling strategy is still present but not such dominant. A big influence of the polarization can be seen which limits the process window due to a negative effect on the circularity $\gamma$.

Also the holes drilled with ps-laser show less taper than those drilled with ns-pules. Therefore and because of the less dominant heat effect the pitch can be chosen smaller for the perforation process with ps-pulses.

\section{Conclusions}

Stable processes are important for micro drilling and structuring as the areas to machine get bigger and bigger. The laser processes have to be stable for hours or even days. Therefore the influencing factors for the stability of micro machining with ps-pulses have to be defined.

Laser percussion drilling of hole arrays is investigated in terms of the hole diameter $d$, ellipticity $\varepsilon$ and circularity $\gamma$. The drilling strategy and the number of pulses per hole are varied using a ps pulsed laser at a wavelength of $355 \mathrm{~nm}$. For the experiment a $15 \mu \mathrm{m}$ thick Aluminum foil is used.

Process for in-line strategy is more stable than for random strategy. The minimal pitch for in-line strategy at a hole diameter of $d_{\min / \max }=5$ resp. $6 \mu \mathrm{m}$ is $a=12 \mu \mathrm{m}$, for random strategy $a=13 \mu \mathrm{m}$. The smalles pitch for a stable process is $a=14 \mu \mathrm{m}$. At this pitch a perforation degree of $\xi$ $=5,7 \%$ for random strategy and $\xi=5 \%$ for in-line strategy is achieved. No further interference between the holes is achieved for pitches $a \geq 19 \mu \mathrm{m}$.

The process for number of pules for the percussion drilling is limited to a small window. Here it is shown to be $150<\mathrm{n}<175$ pulses. For larger numbers of pulses the process is limited by the decrease of the circularity $\gamma$.
The variation of the ellipticity $\varepsilon$ is to account to changes in the heat distribution within the foil for all drilled holes. As for each hole the already drilled pattern changes the heat distribution in which direction the heat can dissipate, the ellipticity varies more than for in-line process.

The circularity $\gamma$ depends on two criteria which limit the process stability. The first is a deformation of the foil material before cooling down due to later drilled holes. The second criterion which influences the circularity is an effect due to polarization which generate linear structures superposing the round taper of the holes.

In contrast to the perforation process with ns-pulses polarization effects play a major role for the perforation process of thin foil with ps-pulses.

\section{Acknowledgment}

Parts of the here depicted research were funded by the German Research Foundation DFG as part of the Cluster of Excellence "Integrative Production Technology for HighWage Countries" and by the Fraunhofer-Gesellschaft within the project RapidDrill.

\section{References}

[1] J. Bekesi, J.J.J. Kaakkunen, W. Michaeli, F. Klaiber, M. Schoengart, J. Ihlemann, and P. Simon, Appl Phys A 99 (2010) p. 691

[2] I. Gehrke. Metallische Mikrosiebe (Logos Verlag, Berlin 2008)

[3] R. Knappe and A. Nebel, Proc. of SPIE, 6871 (2008) 687121

[4] A. Gillner, A. Dohrn and C. Hartmann, 3rd International CIRP High Performance Cutting Conference (2008) p. 199

[5] G. Rutterford, D. Karnakis, A. Webb and M. Knowles, Proceedings of the Advanced Laser Applications Conference (2005)

[6] S. Bruening, G. Hennig, S. Eifel and A. Gillner, Physics Procedia 12 (2011) p. 105

[7] A. Luft, U. Franz, A. Emsermann and J. Kaspar, Appl. Phys. A 63 (1996) p. 93

[8] N. Hambach, C. Hartmann, J. Holtkamp and A. Gillner, Journal of Laser Applications, 24 (2012) 032001

[9] S. Nolte, C. Momma, G. Kamlage, A. Ostendorf, C. Fallnich, F. von Alvensleben and H. Welling, Applied Physics A: Materials Science \& Processing, 68, (1999) p. 563

[10]O.J. Allegre, W. Perrie, K. Bauchert, D. Liu, S.P. Edwardson, G. Dearden and K.G. Watkins, Proceedings of the 36th International MATADOR Conference (2010) p.553

(Received: August 26, 2013, Accepted: November 29, 2013) 